\title{
Discrete Wavelet Transform Based Wireless Digital Communication Systems
}

\author{
Prof Ali A. A. MIEEE, MComSoc \\ Department of Electrical Engineering, Isra University, \\ Amman Jordan
}

\section{Introduction}

There has been paradigm shift in mobile communications systems every decade. Now, just coming into the new century, it might be a good time to start discussions on the fourth generation (4G) systems which may be in service around 2010. For systems beyond 3G, there may be a requirement for a new wireless access technology for the terrestrial components [1]. It's envisaged that these potential new radio interfaces will support up to approximately $100 \mathrm{Mbps}$ for high mobility and up to $1 \mathrm{Gbps}$ for the low mobility, such as nomadic, leads to the $4^{\text {th }}$ generation system. The data rate figures are targets for research and investigation on the basic technologies necessary to implement the vision. The future system specification and the design will be based on the results of the research and investigations.

Due to the high rate requirements, additional spectrum will be needed for the new capabilities beyond International Mobile Telecommunications-2000 (IMT-2000). In conjunction with the future development of IMT-2000 and systems beyond IMT-2000 there will be an increasing relation ship between radio access and communication system, such as wireless Personal Area Networks $\left(\mathrm{PAN}_{\mathrm{s}}\right)$, Local Area Networks $\left(\mathrm{LAN}_{\mathrm{s}}\right)$, digital broadcast, and fixed wireless access.

In discussion about $2 \mathrm{G}$ systems in the 1980, two candidates for the radio access technique existed, Time Division Multiple Access (TDMA) and Code Division Multiple Access (CDMA) schemes. In discussion about 3G system, the Orthogonal Frequency Division Multiplexing (OFDM) appeared in the $1990_{\mathrm{s}}$ and gained a lot of attention and is a potential candidate for $4 \mathrm{G}$ systems. OFDM is very efficient in spectrum usage and is very effective in a frequency selective channel. A variation of OFDM which allows multiple accesses is MultiCarrier CDMA (MC-CDMA) which is essentially an OFDM technique where the individual data symbols are spread using spreading code in frequency domain. The inherent processing gain due to the spreading helps in interference suppression in addition to providing high data rates. OFDM is already the technique used in Digital Audio and Video Broadcasting (DAB, DVB) and $\mathrm{WLAN}_{\mathrm{s}}, 802-11$ family, and is believed to be the technique for future broadband wireless access [2]. The present third generation (3G) systems can provide a maximum data rate of $2 \mathrm{Mbps}$ for indoor environment which is quite less than the needed for the currently evolving multimedia applications requiring very high bandwidth.

This had led the researchers worldwide to the evolution of the 4G systems that are expected to provide a data rate ranging from $20 \mathrm{Mbps}$ to $100 \mathrm{Mbps}$ on the air interface. The reader can easily understand the reason why the OFDM is suited for $4 \mathrm{G}$ systems, with some justifications that appeared as follows: 
Multicarrier techniques can combat hostile frequency selective fading countered in mobile communications. The robustness against frequency selective fading is very attractive, especially for high-speed data transmission [3]

OFDM scheme has been well matured through research and development for high-rate wireless $\mathrm{LAN}_{\mathrm{s}}$ and terrestrial digital video broadcasting.

By combining OFDM with CDMA, it has been synergistic effect, such as enhancement of robustness against frequency selective fading and high scalability in possible data transmission rate.

OFDM can provide higher data rates as is a very good choice for service providers to compete with wire-line carriers [3]. The CDMA scheme is robust to frequency selective fading and has been successfully introduced in commercial cellular mobile communications systems such as Interim Standard-95 (IS-95) and 3G systems. Combining multi-carrier OFDM transmissions with Code Division Multiple Accesses (CDMA) allows us to exploit the wideband channels inherent frequency diversity by spreading each symbol across multiple carriers.

Although OFDM is robust to frequency selective fading, it has severe disadvantages in subcarrier synchronization and sensitivity to frequency offset estimation. The other one is related with the presence of a large number of sub-carriers which exhibit a non-constant nature in its envelope. The combining of OFDM and CDMA has one major advantage though; it can lower the symbol rate in each sub-carrier compared to OFDM so that longer symbol duration makes it easier to synchronize. The MC-CDMA not only mitigates the Inter-Symbol Interference (ISI) but also exploits the multipath. The MC-CDMA suffers only slightly in presence of interference as opposed to Direct Sequence-CDMA (DS-CDMA) whose performance decreases significantly in the presence of interference [4].

In the second section of this chapter, the theory of the Wavelet Transform (with a special concentration on the Discrete Wavelet Transform) will be presented in a very simple and comprehensive manner to make it understandable enough for the formulation of the next sections where the Wavelet based Wireless Digital Communication Systems will be discussed. Also performance comparisons of Fourier and Wavelet based communication systems on different channel models will be presented.

\section{Wavelet transform}

Any general signal can be decomposed into wavelets, i.e., the original function is synthesized by adding elementary building blocks, of constant shape but different size and amplitude. In this approach, one can design a set of basis functions by choosing a proper basic wavelet $\Psi(t)$ (mother wavelet) and use a delayed and scaled version of that. The most important properties of wavelets are the admissibility and the regularity conditions and these are the properties which gave wavelets their name. It can be shown [5] that square integrable functions $\Psi(t)$ satisfying the admissibility condition:

$$
\int \frac{|\Psi(w)|^{2}}{|w|} d w<+\infty
$$

can be used to first analyze and then reconstruct a signal without loss of information. In (1) $\Psi(w)$ stands for the Fourier transform of $\Psi(t)$. The admissibility condition implies that the Fourier transform of $\Psi(t)$ vanishes at the zero frequency, i.e. 


$$
\left.|\Psi(w)|^{2}\right|_{w=0}=0
$$

A zero at the zero frequency also means that the average value of the wavelet in the time domain must be zero and therefore it must be oscillatory. In other words, $\Psi(w)$ must be a wave. The reconstruction or inverse transformation is satisfied whenever $\Psi(w)$ is of finite energy and band pass (oscillates in time like a short wave). These are the regularity conditions and they state that the wavelet function should have some smoothness and concentration in both time and frequency domains. For sufficiently $\operatorname{regular} \Psi(w)$, the reconstruction condition is:

$$
\int \Psi(t) d t=0
$$

Summarizing, the admissibility condition gave us the wave, regularity and vanishing moments gave us the fast decay or the let, and together they give us the wavelet.

\subsection{The discrete wavelet transform}

Under the reconstruction condition (3), the continuously labeled basis functions (wavelets), $\Psi_{j, k}(t)$ behaves in the wavelet analysis and synthesis just like an orthonormal basis. By appropriately discretizing the time-scale parameters, $\tau, \mathrm{s}$, and choosing the right mother wavelet, $\Psi(t)$, it is possible to obtain a true orthonormal basis. The natural way is to discretizing the scaling variable $s$ in a logarithmic manner $\left(s=s_{0}^{-j}\right)$ and to use Nyquist sampling rule, based on the spectrum of function $x(t)$, to discretizing $\tau$ at any given scale $\left(\tau=k s_{0}^{-j} T\right)$. The resultant wavelet functions are then as follows:

$$
\Psi_{j, k}(t)=s_{0}^{j / 2} \Psi\left(s_{0}^{j} t-k \tau_{0}\right)
$$

If $\mathrm{s} 0$ is close enough to one and if $\mathrm{T}$ is small enough, then the wavelet functions are overcomplete and signal reconstruction takes place within non-restrictive conditions on $\Psi(t)$. On the other hand, if the sampling is sparse, e.g., the computation is done octave by octave $(\mathrm{s} 0=0)$, a true orthonormal basis will be obtained only for very special choices of $\Psi(t)$. Based on the assumption that wavelet functions are orthonormal:

$$
\int \Psi_{j, k}(t) \Psi_{m, n}(t) d t=\left\{\begin{array}{cc}
1 & \text { if } j=m \text { and } k=n \\
0 & \text { otherwise }
\end{array}\right.
$$

For discrete time cases, equation (4) is generally used with $\mathrm{s} 0=2$, the computation is done octave by octave. In this case, the basis for a wavelet expansion system is generated from simple scaling and translation. The generating wavelet or mother wavelet, represented by $\Psi(t)$, results in the following two-dimensional parameterization of $\Psi_{j, k}(t)$.

$$
\Psi_{j, k}(t)=2^{j / 2} \Psi\left(2^{j} t-k\right)
$$

The $2 \mathrm{j} / 2$ factor in equation (6) normalizes each wavelet to maintain a constant norm independent of scale $\mathrm{j}$. In this case, the discretizing period in $\tau$ is normalized to one and is 
assumed that it is the same as the sampling period of the discrete $\operatorname{signal}\left(\tau=k 2^{-\mathrm{j}}\right)$. All useful wavelet systems satisfy the multiresolution conditions. In this case, the lower resolution coefficients can be calculated from the higher resolution coefficients by a treestructured algorithm called filter-bank [6]. In wavelet transform literatures; this approach is referred to as discrete wavelet transform (DWT).

\subsubsection{The scaling function}

The multiresolution idea is better understood by using a function represented by $\Phi(t)$ and referred to as scaling function. A two-dimensional family of functions is generated, similar to (6), from the basic scaling function by [7]:

$$
\Phi_{j, k}(t)=2^{j / 2} \Phi\left(2^{j} t-k\right)
$$

Any continuous function, $f(t)$, can be represented, at a given resolution or scale j0, by a sequence of coefficients given by the expansion:

$$
f_{j_{0}}(t)=\sum_{k} f_{j_{0}}[k] \cdot \Phi_{j_{0}, k}(t)
$$

In other words, the sequence $x_{j_{0}}[k]$ is the set of samples of the continuous function $\mathrm{x}(\mathrm{t})$ at resolution $\mathrm{j} 0$. Higher values of $\mathrm{j}$ correspond to higher resolution. Discrete signals are assumed samples of continuous signals at known scales or resolutions. In this case, it is not possible to obtain information about higher resolution components of that signal. The main required property is the nesting of the spanned spaces by the scaling functions. In other words, for any integer $\mathrm{j}$, the functional space spanned by [8]:

$$
\left\{\Phi_{j, k}(t) ; \text { for } k \in 1,2, \ldots\right\}
$$

should be a subspace of the functional space spanned by:

$$
\left\{\Phi_{j+1, k}(t) ; \text { for } k \in 1,2, \ldots\right\}
$$

The nesting of the space spanned by $\Phi\left(2^{j} t-k\right)$ is achieved by requiring that $\Phi(t)$ be represented by the space spanned by $\Phi(2 t)$. In this case, the lower resolution function, $\Phi(t)$, can be expressed by a weighted sum of shifted version of the same scaling function at the next higher resolution, $\Phi(2 t)$, as follows:

$$
\Phi(t)=\sum_{k} h(k) \sqrt{2} \Phi(2 t-k)
$$

The set of coefficients $h(k)$ being the scaling function coefficients and $\sqrt{2}$ maintains the norm of the scaling function with scale of two. $\Phi(t)$ being the scaling function which satisfies this equation which is sometimes called the refinement equation, the dilation equation, or the multiresolution analysis equation (MRA). 


\subsubsection{The wavelet functions}

The important features of a signal can better be described or parameterized, not by using $\Phi_{j, k}(t)$ and increasing $\mathrm{j}$ to increase the size of the subspace spanned by the scaling functions, but by defining a slightly different set of functions $\Psi_{j, k}(t)$ that span the differences between the spaces spanned by the various scales of the scaling function.

Since it is assumed that these wavelets reside in the space spanned by the next narrower scaling function, they can be represented by a weighted sum of shifted version of the scaling function $\Phi(2 t)$ as follows:

$$
\Psi(t)=\sum_{k} g(k) \sqrt{2} \Phi(2 t-k)
$$

The set of coefficients $g(k)$ 's is called the wavelet function coefficients (or the wavelet filter). It is shown that the wavelet coefficients are required by orthogonality to be related to the scaling function coefficients by $[9,10]$ :

$$
g(k)=(-1)^{n} h(1-k)
$$

One example for a finite even length-N $h(k)$

$$
g(k)=(-1)^{k} h(N-1-k)
$$

The function generated by equation (12) gives the prototype or mother wavelet $\Psi(t)$ for a class of expansion functions of the form shown in equation (6). For example the Haar scaling function is the simple unit-width, unit-height pulse function $\Phi(t)$ shown in Fig (1a) [7] and it is obvious that $\Phi(2 t)$ can be used to construct $\Phi(t)$ by:

$$
\Phi(t)=\Phi(2 t)+\Phi(2 t-1)
$$

Which means (11) is satisfied for coefficients $h(0)=1 / \sqrt{2}, h(1)=1 / \sqrt{2}$.

The Haar wavelet function that is associated with the scaling function in Fig.(1a) is shown in Fig. (1b). For Haar wavelet, the coefficients in equation (14) are $g(0)=1 / \sqrt{2}, g(1)=-1 / \sqrt{2}$.

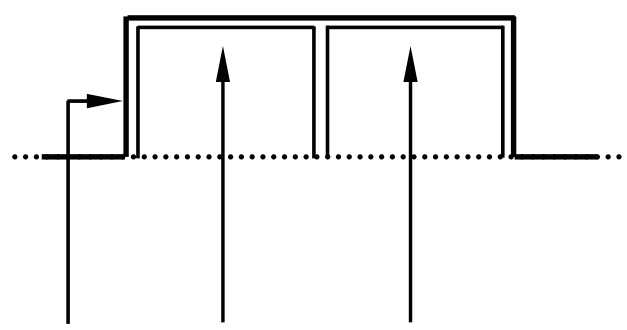

$$
\Phi(t)=\Phi(2 t)+\Phi(2 t-1)
$$

(a)

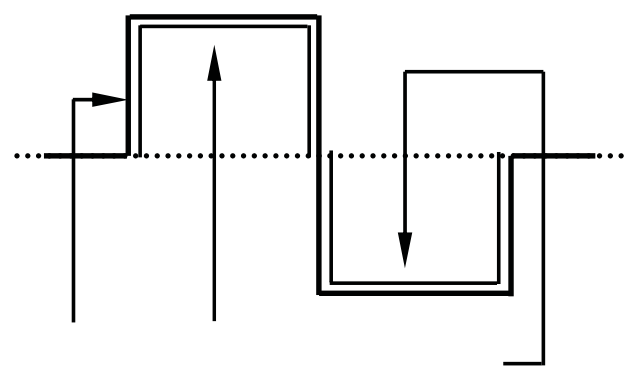

$$
\Psi(t)=\Phi(2 t)-\Phi(2 t-1)
$$

(b)

Fig. 1. (a) Haar Scaling Function, (b) Haar wavelet function. 
Any function $f(t)$ could be written as a series expansion in terms of the scaling function and wavelets by [11]:

$$
f(t)=\sum_{k=-\infty}^{\infty} a_{j_{0}}(k) \Phi_{j_{0}, k}(t)+\sum_{j=j_{0}}^{\infty} \sum_{k=-\infty}^{\infty} b_{j}(k) \Psi_{j, k}(t)
$$

In this expansion, the first summation gives a function that is a low resolution or coarse approximation of $f(t)$ at scale $j 0$. For each increasing $j$ in the second summation, a higher or finer resolution function is added, which adds increasing details. The choice of $\mathrm{j} 0$ sets taking the coarsest scale whose space is spanned by $\Phi_{j_{0} . k}(t)$. The rest of the function is spanned by the wavelets providing the high-resolution details of the function. The set of coefficients in the wavelet expansion represented by equation (14) is called the discrete wavelet transform (DWT) of the function $\mathrm{f}(\mathrm{t})$.

These wavelet coefficients, under certain conditions, can completely describe the original function, and in a way similar to Fourier series coefficients, can be used for analysis, description, approximation, and filtering. If the scaling function is well behaved, then at a high scale, samples of the signal are very close to the scaling coefficients. As mentioned before, for well-behaved scaling or wavelet functions, the samples of a discrete signal can approximate the highest achievable scaling coefficients.

It is shown that the scaling and wavelet coefficients at scale $j$ are related to the scaling coefficients at scale $(j+1)$ by the following two relations.

$$
\begin{aligned}
& a_{j}(k)=\sum_{m} h(m-2 k) a_{j+1}(m) \\
& b_{j}(k)=\sum_{m} g(m-2 k) b_{j+1}(m)
\end{aligned}
$$

\subsection{Fast computation method of DWT}

The implementation of equations (17) and (18) is illustrated in Fig. (2). In this figure, two levels of decomposition are depicted. $\mathrm{h}$ and $\mathrm{g}$ are low-pass and high-pass filters corresponding to the coefficients $h(n)$ and $g(n)$ respectively. The down-pointing arrows denote a decimation or down-sampling by two. This splitting, filtering and decimation can be repeated on the scaling coefficients to give the two-scale structure. The first stage of two banks divides the spectrum of $a_{j-1, k}$ into a low-pass and high-pass band, resulting in the scaling coefficients and wavelet coefficients at lower scale $a_{j, k}$ and $b_{j, k}$. The second stage then divides that low-pass band into another lower low-pass band and a high-pass filter.

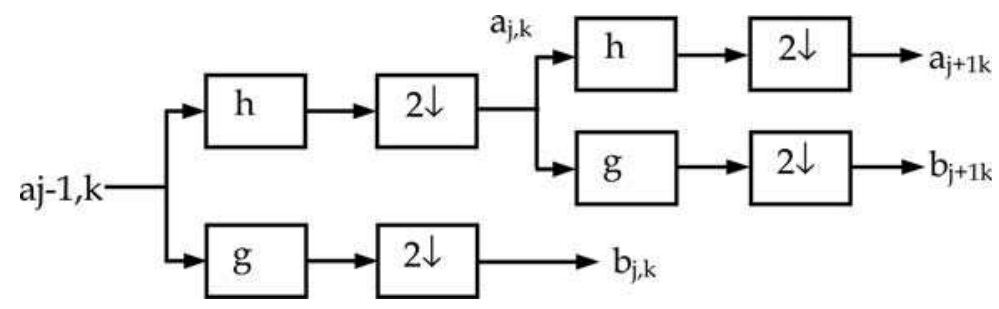

Fig. 2. The filter bank for calculating the wavelet coefficients. 


\section{OFDM and CDMA theory and concept}

The purpose of this section is to describe the OFDM and CDMA systems. The majority of the information presented is intended as background information for the system simulation and evaluation presented in rest of the chapter. The basic theoretical aspects for mobile radio channels with a brief mathematical explanation of multi-path selective channels and their characteristic parameters are explored, due to their direct effect on the performance of the OFDM and CDMA modulation techniques. Then the FFT-based OFDM and different combinations of MC and CDMA are given together with their performances and simulation. Then it provides the basic theory of used transforms, wavelet, and its equations and a basic outlines for its computation algorithm using D4 wavelet. Also Multi-wavelets theory with its equations is introduced.

\subsection{Orthogonal frequency division multiplexing (OFDM)}

A simple multi-carrier communication system is the frequency division multiplexing (FDM) or multi-tone. The broad transmission bandwidth is divided into many narrow nonoverlapping sub-channels, in which the data is transmitted in a parallel fashion. Ideally each sub-channel is narrow enough so that the sub-channel can be considered to be slow, flat fading to reduce the effect of ISI. The fundamental structure of a multi-carrier system is depicted in Fig. (3). The data stream is mapped to the desired waveform, filter banks are used to limit the signal bandwidth. After being modulated by separate center frequencies, these signals are multiplexed and transmitted. At the receiver the frequency multiplexed signal is down converted to different channels by multiplication with separate center frequencies, filtered by the filter banks to get the base band Multicarrier signal for further processing.

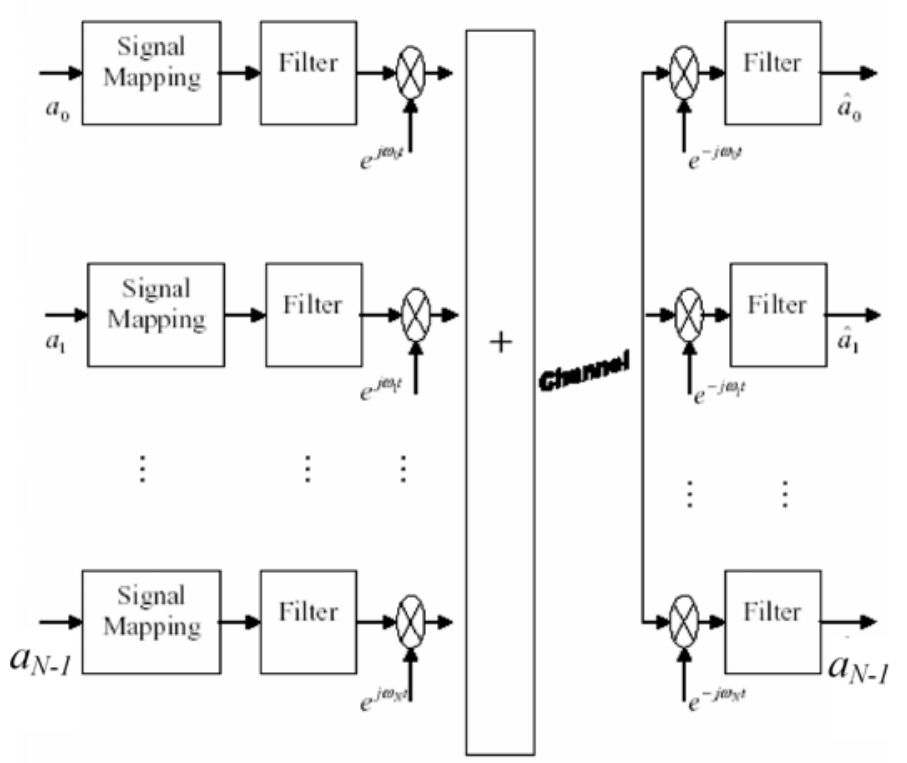

Fig. 3. Fundamental structure of a multi-carrier transceiver-carrier 


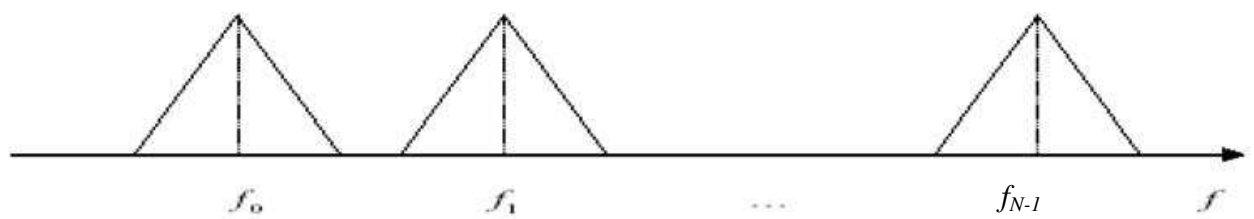

Fig. 4. FDM sub-bands spectrum distribution.

The spectrum allocation for sub-channels in a FDM system is shown in Fig (4), where f0, f1, $\ldots \ldots, \mathrm{fN}-1$ are the center frequencies of the sub-channels.

This modulation has the following disadvantages:

1. Since the sub-carriers are not overlapped with each other, the wide spacing between the sub-bands means lower spectrum efficiency.

2. The complexity will increase due to the $\mathrm{N}$ modulators and filters used at the transmitter and $\mathrm{N}$ demodulators and filters at the receiver which makes the system more complicated [13]

\subsubsection{Implementation of OFDM signals}

For Fig. (3), with input sequence $\{a[k]\}, 0 \leq \mathrm{k} \leq \mathrm{N}-1$, the frequency spacing $\square \Delta \mathrm{f} \quad \square$ between the different sub-carriers and the symbol interval $\mathrm{Tu}$, the transmitted data can be expressed as:

$$
x_{a}(t)=\sum_{k=0}^{N-1} a[k] e^{j 2 \pi k \Delta f t}, \quad 0 \leq \mathrm{t} \leq \mathrm{Tu}
$$

If the signal is sampled at a rate $\mathrm{Tu} / \mathrm{N}$, then the above equation can be rewritten as:

$$
x_{a}[n]=x_{a}\left(\frac{n}{N} T_{u}\right)=\sum_{k=0}^{N-1} a[k] e^{j 2 \pi k \Delta f T_{u} / N}
$$

If the following equation:

$$
\Delta \mathrm{f} \mathrm{Tu}=1 \quad\left(\Delta \mathrm{f}=\frac{1}{\mathrm{Tu}}\right)
$$

is satisfied, then the multi-carriers are orthogonal to each other and equation (20) can be rewritten as:

$$
x_{a}[n]=\sum_{k=0}^{N-1} a[k] e^{j 2 \pi n k / N}
$$

One of the major advantages of OFDM is that the modulation can be performed in the discrete domain using an Inverse Discrete Fourier Transform (IDFT) or more computationally efficient inverse Fast Fourier Transform (IFFT). The above equation is just the IDFT of the input signal stream $\{a[k]\}$, equation (22) can be rewritten as [14]:

$$
X_{e}[n]=N . I D F T\{a[k]\}
$$


At the receiver the DFT implementation to find the approximate signal $\mathrm{a}[\mathrm{k}]$ can be written as:

$$
\begin{aligned}
& \hat{a}[k]=\operatorname{DFT}\left\{x_{a}[n]\right\} \\
& =\sum_{n=0} x_{a}[n] e^{-j 2 \pi n k / N} \\
& =\frac{1}{N} \sum_{n=0}^{N-1} \sum_{m=0}^{N-1} a[m] e^{j 2 \pi n(m-k) / N} \\
& =\frac{1}{N} \sum_{m=0}^{N-1} a[m] \sum_{n=0}^{m=0} e^{j 2 \pi n(m-k) / N}=\frac{1}{N} \sum_{m=0}^{N-1} a[m] N \delta[m-k] \\
& =a[k]
\end{aligned}
$$

Here $\delta[\mathrm{m}-\mathrm{k}]$ is the delta function defined as :

$$
\delta[n]\left\{\begin{array}{l}
1, \text { if } n=0 \\
0, \text { otherwise }
\end{array}\right.
$$

From the derivation above, it can be observed that there are two most important features of the OFDM technique, these are:

1. Each sub-carrier has a different center frequency. These frequencies are chosen so that the following integral over a symbol period is satisfied:

$$
\int_{0}^{T u} a_{m} e^{j w_{m} t} a_{l} e^{j w_{l} t} d t=0, m \neq l
$$

The sub-carrier signals in an OFDM system are mathematically orthogonal to each other. The sub-carrier pulse used for transmission is chosen to be rectangular. The rectangular pulse leads to a $\frac{\sin (x)}{x}$ type of spectrum. The spectrum of the three adjustment OFDM sub-carriers is illustrated in Fig. (5). The spectrum of the sub-carriers is overlapped to each other, thus the OFDM communication system has high spectrum efficiency. Maintenance of the orthogonality of the sub-carriers is very important in an OFDM system, which requires the transmitter and receiver to be in the perfect synchronization [12].

2. IDFT and DFT functions can be exploited to realize the OFDM modulation and demodulation instead of the filter banks in the transmitter and the receiver to lower the system implementation complexity and cost. This feature is attractive for practical use. The IFFT and FFT algorithms can be used to calculate the IDFT and DFT efficiently. IFFT and FFT are used to realize the OFDM modulation and demodulation to reduce the system implementation complexity and to improve the system running speed. 


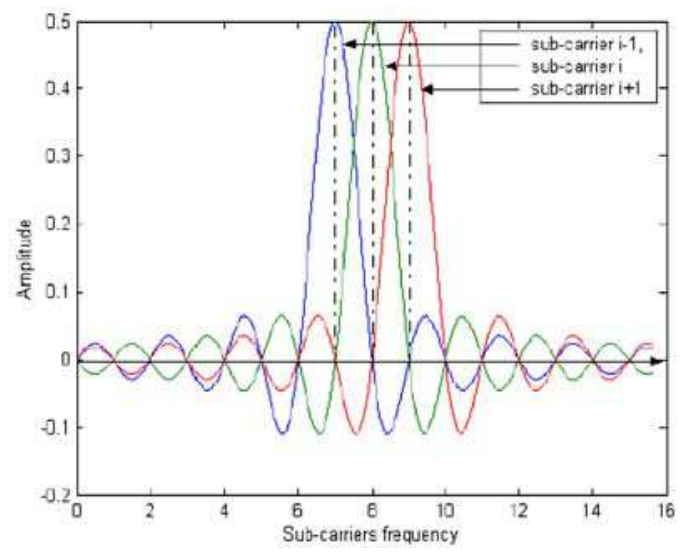

Fig. 5. Orthogonality principle of OFDM

It is necessary for a predetermined number of sub symbols to be available simultaneously at the inputs of the IFFT unit. For this reason the sequentially received data are temporarily stored, until the required number of sub symbols for parallel transmission have accumulated, and are then read out in parallel.

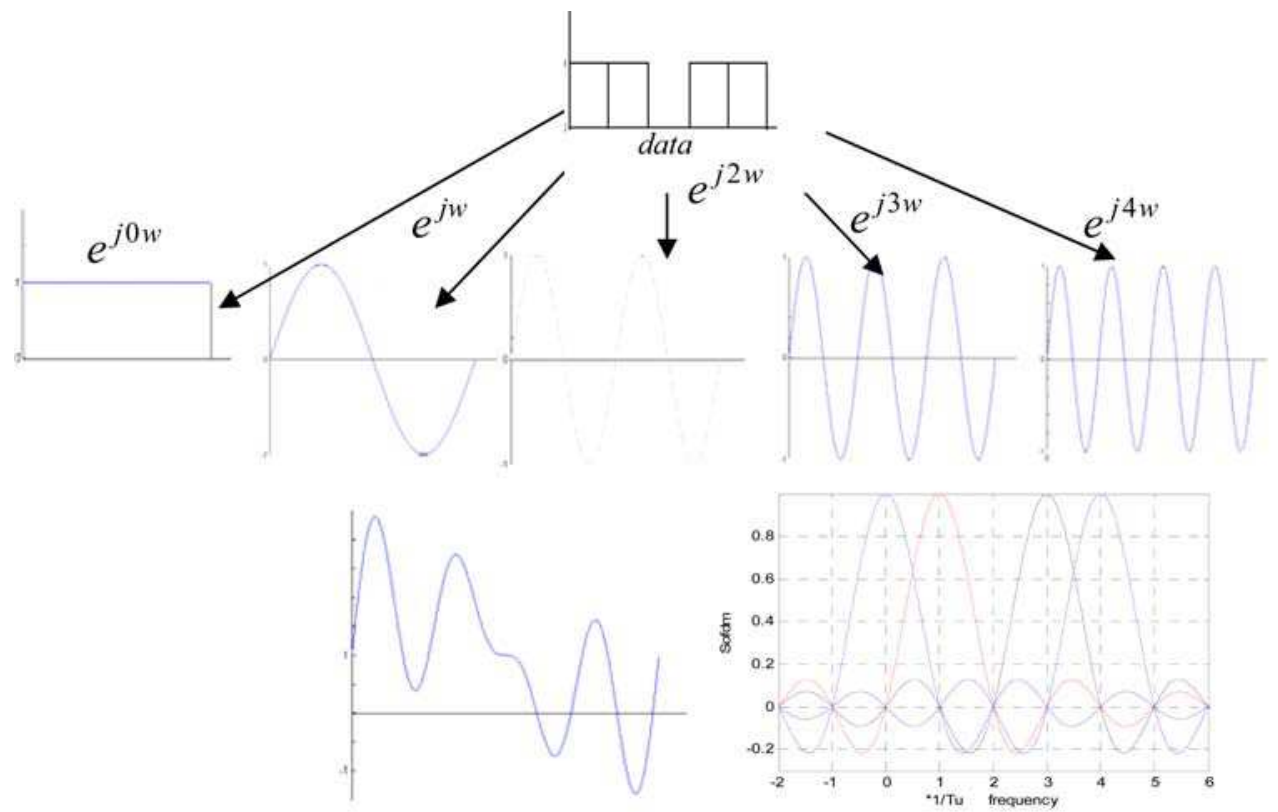

Time domain-OFDM signal Frequency domain-OFDM signal

Fig. 6. Signal processing of OFDM

Fig.(6) above demonstrates by a simple example the principle of the signal processing within the subsequent IFFT unit. In this example an OFDM symbol is shaped from five consecutive 
bits. The first diagram represents the serial data stream. After the parallel transformation each bit lies at one of the inputs of the IFFT unit for the duration $\mathrm{Tu}=5 \mathrm{~Tb}$ and generates a sub signal. The frequencies of the individual sub signals result in integral multiples of $f 0$ $=1 / \mathrm{Tu}$. They are therefore orthogonal to one another [15]

\subsubsection{Guard Interval}

One of the most important properties of OFDM transmissions is the robustness against multipath delay spread. This is achieved by having a long symbol period, which minimizes the inter-symbol interference. The level of robustness can in fact be increased even more by the addition of a guard period between transmitted symbols. The guard period allows time for multipath signals from the pervious symbol to die away before the information from the current symbol is gathered [16].

As long as the multipath delay echoes stay within the guard period duration, there is strictly no limitation regarding the signal level of the echoes: they may even exceed the signal level of the shorter path. The signal energy from all paths just adds at the input to the receiver, and since the FFT is energy conservative, the whole available power feeds the decoder. If the delay spread is longer then guard intervals then they begin to cause inter symbol interference. However, provided the echoes are sufficiently small they do not cause significant problems. This is true most of the time as multipath echoes delayed longer than the guard period will have been reflected off very distant objects. There are several types of guard interval such that cyclic prefix $(\mathrm{CP})$, zero padded, and other variation of guard interval are possible.

\subsubsection{Cyclic prefix}

The most effective guard period to use is a cyclic extension of the symbol, see Fig (7). If a mirror in time, of the end of the symbol waveform is put at the start of the symbol as the guard period, this effectively extends the length of the symbol, while maintaining the orthogonality of the waveform. Using this cyclic extended symbol the samples required for performing the FFT can be taken anywhere over the length of the symbol. This provides multipath immunity as well as symbol time synchronization tolerance.

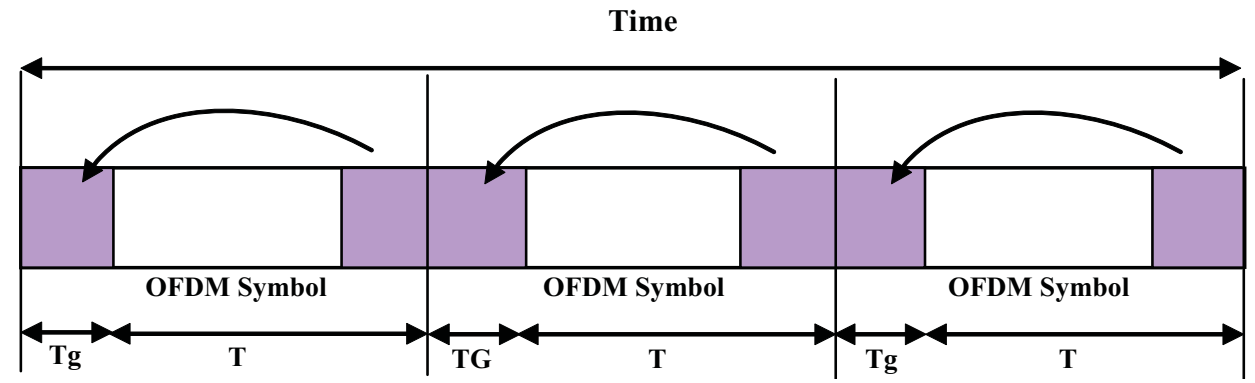

Fig. 7. Cyclic extension of OFDM transmitted symbol tation of OFDM

\subsubsection{Zero padding}

Another type of guard interval is a zero-padding .Instead of inducing the cyclic prefix, each IFFT processed block is zero padded, by many zeros depending on channel's order to 
eliminate ISI. If the number of zeros padded is equal to cyclic prefix length, then ZP-OFDM and CP-OFDM transmission has the same spectral efficiency.

Other types of guard intervals are possible. One possible type is to have half the guard period a cyclic prefix of the symbol, as in cyclic prefix type, and the other half a zero padded, as above [16].

\subsection{Synchronization of OFDM systems}

Synchronization is a big hurdle in OFDM. Synchronization usually consists of three parts as follows:

\subsubsection{Frame detection}

Frame detection is used to determine the symbol boundary so that correct samples for a symbol frame can be taken. The sampling starting point TX at the receiving end must satisfy the condition $\tau_{\max }\left\langle T_{x}\left\langle T_{g}\right.\right.$, where $\tau_{\max }$ is maximum delay spread. Since the previous symbol will only have effect over samples within [0,tmax],there is no ISI [18].

There are many algorithms that can be applied to estimate the start of an OFDM symbol based on pilots or on the cyclic prefix. A good synchronization method must be fast, have a reliable indication of the synchronized state and introduce a minimum of redundancy in the transmitted stream.

Most existing timing algorithms use correlations between repeated OFDM signal portions to create a timing plateau. Such algorithms are not able to give precise timing position especially when the SNR is low. To improve the robustness of the algorithms, in [29] they used a differentially coded time-domain PN sequence for frame detection. Because of its delta like self-correlation property, the PN sequence allows to find the precise timing position. The PN sequence is transmitted as part of the OFDM packet preamble. At the receiver, the received signal samples are correlated with the known sequence. When the transmitted PN sequence is aligned with receiver PN sequence, a correlation peak is observed from which the OFDM symbol boundary can be inferred.

\subsubsection{Carrier synchronization error}

Carrier frequency offset estimation plays an important role in OFDM communication systems because of their high sensitivity to carrier frequency offsets [19]. Due to the carrier frequency difference of the transmitter and receiver, each signal sample at time $t$ contains an unknown phase factor $e^{j 2 \pi \Delta f_{c} t}$, where $\Delta \mathrm{f}$ is the unknown carrier frequency offset. This unknown phase factor must be estimated and compensated for each sample before FFT at the receiver, since otherwise the orthogonality between sub-carriers is lost.

The impact of a frequency error can be seen as an error in the frequency instants, where the received signal is sampled during demodulation by the FFT Fig (8) depicts this two-fold effect. The amplitude of the desired sub-carrier is reduced (' + ') and inter-carrier-interference ICI arises from the adjacent sub-carriers ('0’) [20].

\subsubsection{Sampling error correction}

Because the sampling clock difference between the transmitter and receiver, each signal sample is off from its correct sampling time by a small amount which is linearly increasing with the index of the sample. For example, for $100 \mathrm{ppm}$ crystal offset, it will be off by 1 


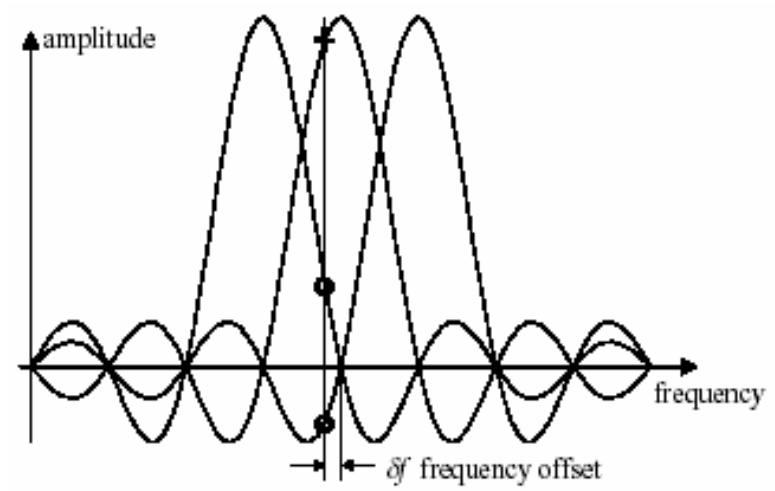

Fig. 8. Inter-carrier-interference (ICI) arises in case of a carrier synchronization error.

sample after 10000 samples. If a symbol contains 100 samples, then within each symbol the maximum offset will be $1 \%$ of a sample. Although this may cause the orthogonality degrading between the sub-carriers, it can usually be ignored. If sampling error must be corrected, then interpolation filter must be used to construct the signal at correct sampling time [18].

\section{Mobile radio channels}

In mobile radio channels, the transmitted signal suffers from different effects, which are characterized as follows [21],[22],[23]:

Multi-path propagation occurs as a consequence of reflection, scattering, and diffraction of the transmitted electromagnetic wave at natural and man-made objects. Thus, at the receiver antenna, a multitude of waves arrives from many different directions with different delays, attenuations, and phases as shown in Fig.(9), which shows that the terminal station (TS) may receive the direct signal from the base station (BS) as well as several signals generated due to reflections and scattering. The superposition of these waves results in amplitude and phase variations of the composite received signal.

Changes in the phases and amplitudes of the arriving waves occur, which lead to timevariant multi-path propagation. Even small movements on the order of the wavelength may result in a totally different wave superposition The varying signal strength due to timevariant multi-path propagation is referred to as fast fading. Shadowing is caused by obstruction of the transmitted waves by e.g., hills, buildings, wall, and trees, which results in more or less strong attenuation of the signal strength. Compared to fast fading, longer distances have to be covered to significantly change the shadowing constellation. The varying signal strength due to shadowing is called slow fading and can be described by a log-normal distribution

Path loss indicates how the mean signal power decays with distance between transmitter, and receiver. In free space, the mean signal power decreases with the square of the distance between (BS) and (TS). In a mobile radio channel, where often no line of site (LOS) path exists, signal power decreases with a power higher than two and is typically in the order of three to five. Variations of the received power due to shadowing and path loss can be efficiently counteracted by power control. 


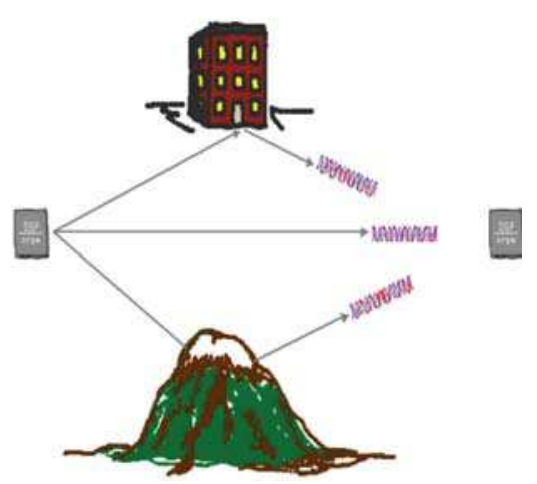

Fig. 9. Composite received signal due to reflections in mobile radio channel

\subsection{Channel modeling}

The mobile radio channel can be characterized by the time-variant channel impulse response $h(\tau, t)$ [24]. The channel impulse response represents the response of the channel at time $t$ due to an impulse applied at time $t-t$. The mobile radio channel is assumed to be a wide-sense stationary random process, i.e., the channel has a fading statistic that remains constant over short periods of time or small spatial distances. In environments with multipath propagation, the channel impulse response is composed of a large number of scattered impulses received over $\mathrm{Np}$ different paths,

$$
h(\tau, t)=\sum_{p=0}^{N_{p}-1} a_{p} e^{j\left(2 \pi f_{d, p^{t}}+\varphi_{p}\right)} \delta\left(\tau-\tau_{p}\right)
$$

where,

$$
\delta\left(\tau-\tau_{p}\right)= \begin{cases}1, & \text { if } \tau=\tau_{p} \\ 0, & \text { otherwise }\end{cases}
$$

and $a_{p}, f_{d, p}, \varphi_{p}$, and $\tau_{p}$ are the amplitude, the Doppler frequency, the phase, and the propagation delay, respectively, associated with the path $p, p=0,1,2, \ldots \ldots \ldots \ldots \ldots, N p-1$. A channel impulse response with corresponding channel transfer function is illustrated in Fig.(10), while, Fig.(11), is a block diagram representation of a fading channel with two paths, i.e., with two rays.
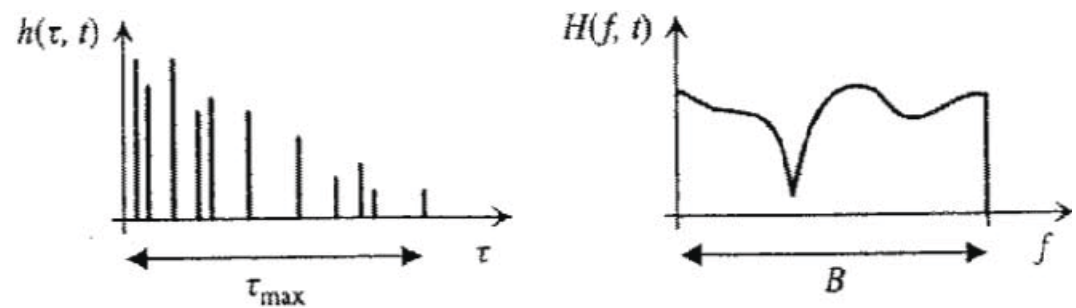

Fig. 10. Time-variant channel impulse response and channel transfer function with frequency-selective fading 
The assigned channel transfer function is

$$
H(f, t)=\sum_{p=0}^{N_{p}-1} a_{p} e^{j\left(2 \pi\left(f_{d, p^{t}}+f \tau_{p}\right)+\varphi_{p}\right)}
$$

The delays are measured relative to the first detectable path at the receiver. The Doppler frequency is given by [22]:

$$
f_{d, p}=\frac{v f_{c} \cos \left(\theta_{p}\right)}{c}
$$

It is obvious that $f_{d, p}$ depends on the velocity $v$ of the terminal station, the speed of light $c$, the carrier frequency $f_{c}$, and the angle of incidence $\theta p$ of a wave assigned to a path $p$.

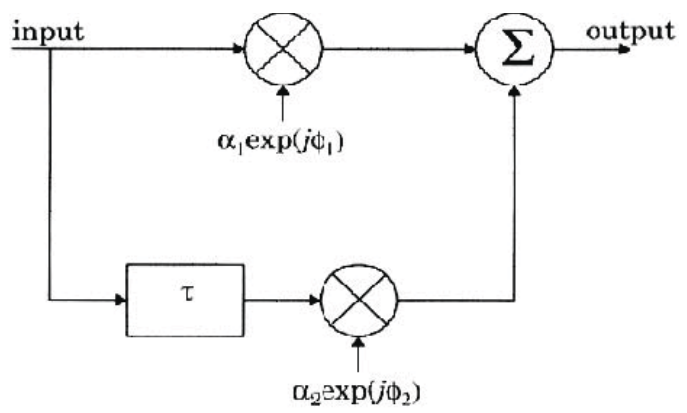

Fig. 11. 2-Ray fading channel

Fig. (12) illustrates the Doppler effect. The delay power density spectrum $\rho(\tau)$ that characterizes the frequency selectivity of the mobile radio channel gives the average power of the channel output as a function of the delay, $\tau$. The mean delay $\tau$, the root mean square (RMS) delay spread tRMS and the maximum

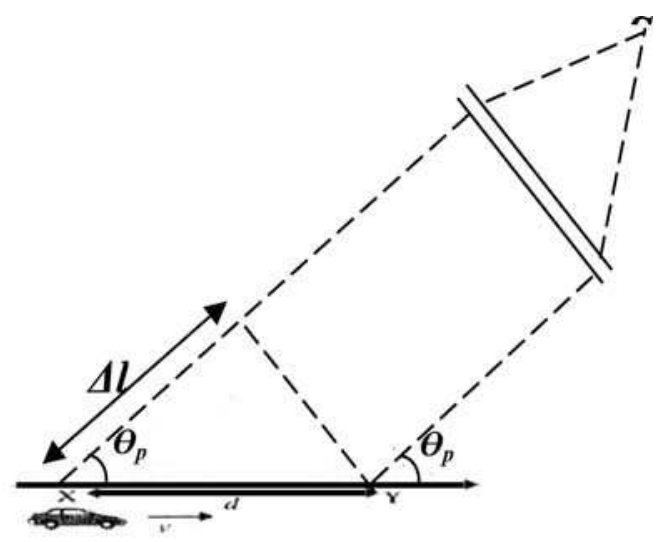

Fig. 12. Illustration of Doppler Effect 
Delay tmax are characteristic parameters of the delay power density spectrum. The mean delay is:

$$
\bar{\tau}=\frac{\sum_{P=0}^{N_{P}-1} \tau_{P}\left|a_{P}\right|^{2}}{\sum_{P=0}^{N_{P}-1}\left|a_{P}\right|^{2}}
$$

where, the term $\left|a_{P}\right|^{2}$, in equation (31) represents the power of path $\mathrm{p}$. The RMS delay spread is defined as:

$$
\tau_{P}=\sqrt{\frac{\sum_{P=0}^{N_{P}-1} \tau_{P}{ }^{2}\left|a_{P}\right|^{2}}{\sum_{P=0}^{N_{P}-1}\left|a_{P}\right|^{2}}-(\bar{\tau})^{2}}
$$

Similarly, the Doppler power density spectrum $\mathrm{S}(\mathrm{fd})$ can be defined as that characterizing the time variance of the mobile radio channel and gives the average power of the channel output as a function of the Doppler frequency fD. The frequency dispersive properties of multi-path channels are most commonly quantified by the maximum occurring Doppler frequency fD max and the Doppler spread fDspread. The Doppler spread is the bandwidth of the Doppler power density spectrum and can take on values up to two times $|\mathrm{fDmax}|[25]$, i.e.,

$$
\text { fDspread } \leq 2|\mathrm{fDmax}|
$$

\subsection{Channel fade statistics}

The statistics of the fading process characterize the channel and are of importance for channel model parameter specifications. A simple and often used approach is obtained from the assumption that there is a large number of scatterers in the channel that contribute to the signal at the receiver side. The application of the central limit theorem leads to a complexvalued Gaussian process for the channel impulse response. In the absence of LOS or a dominant component, the process is zero-mean. The magnitude of the corresponding channel transfer function:

$$
a=a(f, t)=|H(f, t)|
$$

is the random variable, with Rayleigh's distribution given by:

$$
p(a)=\frac{2 a}{\Omega} e^{-\frac{a^{2}}{\Omega}}
$$

where the average power is:

$$
\Omega=\left\{a^{2}\right\}
$$


The phase is uniformly distributed in the interval $[0,2 \pi]$. In the case that the multi-path channel contains a LOS or dominant component in addition to the randomly moving scatters. The channel impulse response can no longer be modeled as zero-mean. Under the assumption of a complex-valued Gaussian process for the channel impulse response, the magnitude of the channel transfer function has a Rice distribution given by:

$$
P(a)=\frac{2 a}{\Omega} e^{-\left(a^{2} / \Omega+K_{\text {Rice }}\right)} I_{0}\left(2 a \sqrt{\frac{K_{\text {Rice }}}{\Omega}}\right)
$$

The Rice factor KRice is determined by the ratio of the power of the dominant path to the power of the scattered paths. Io is the zero-order modified Bessel function. The phase is uniformly distributed in the interval $[0,2 \pi]$.

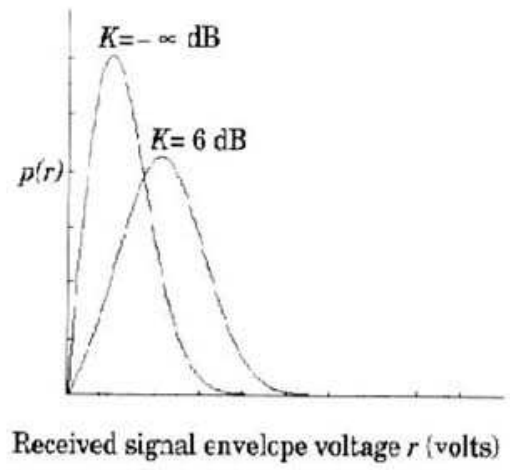

Fig. 13. Probability density function of Ricean Distribution, $k=-\infty$ for (Rayleigh)

\subsection{Inter-symbol interference (ISI) and inter-channel interference (ICI)}

The delay spread can cause inter-symbol-interference (ISI), when adjacent data symbols overlap and interfere with each other due to different delays on different propagation paths. The number of interfering symbols in a single-carrier modulated system is given by

$$
N_{I S I, \text { sin gle-carrier }}=\left\lceil\frac{\tau_{\max }}{T_{d}}\right\rceil
$$

For high data rate applications with very short symbol duration $\mathrm{Td}<\mathrm{\tau max}$, the effect of ISI and, with that, the receiver complexity can increase significantly. The effect of ISI can be counteracted by different measures such as time or frequency domain equalization.

In spread spectrum systems, rake receivers with several arms are used to reduce the effect of ISI by exploiting the multi-path diversity such that individual arms are adapted to different propagation paths.

If the duration of the transmitted symbol is significantly larger than the maximum delay Td>>tmax, the channel produces a negligible amount of ISI. This effect is exploited with multi-carrier transmission where the duration of the transmitted symbol increases with the number of sub-carrier Nc and, hence, the amount of ISI decreases. The number of the interfering symbols in a multi-carrier modulated system is given by: 


$$
N_{I S I, \text { multicarrier }}=\left\lceil\frac{\tau_{\max }}{N_{c} T_{d}}\right\rceil
$$

Residual ISI can be eliminated by the use of a guard interval. The maximum Doppler spread in mobile radio applications using single-carrier modulation is typically much less than the distance between adjacent channels, such that the effect of interference on adjacent channels due to Doppler spread is not a problem for a single-carrier modulated systems. For multicarrier modulated systems, the sub-channel spacing Fs can become quite small, such that Doppler effects can cause significant ICI. As long as all sub-carriers are affected by a common Doppler shift fd, this Doppler shift can be compensated for in the receiver and ICI can be avoided. However, if Doppler spread on the order of several percent of the subcarrier spacing occurs. ICI may degrade the system performance significantly. To avoid performance degradations due to ICI more complex receivers with ICI equalization should be used. The sub-carrier spacing Fs should be chosen as:

$$
F_{S}>f_{D \max }
$$

such that the effect due to Doppler spread can be neglected.

Nevertheless, if a multi-carrier system design is chosen such that the Doppler spread is on the order of the sub-carrier spacing or higher, a rake receiver in the frequency domain can be used. With the frequency domain rake receiver each branch of the rake resolves a different Doppler frequency.

\section{Code division multiple access scheme}

In the context of broadband wireless communications using CDMA without the assistance of frequency/ time hopping, the main multiple access options include Multi-tone CDMA (MT-CDMA) using time domain DS spreading [26], Multicarrier CDMA (MC-CDMA) using frequency domain spreading, as well as Multicarrier DS-CDMA (MC DS-CDMA) using time domain DS spreading of the individual sub-carrier signals [27].

The behavior of the above three CDMA schemes will be investigated when communicating over broadband wireless channels. It will be shown that regardless of the communication environments encountered, both Multi-tone DS-CDMA and MC-CDMA exhibit more severe problems than MC DS-CDMA. Broad-band MC DS-CDMA augmented by transmit diversity is capable of mitigating the problems imposed by broadband wireless channels. It is shown that by appropriately selecting the system parameters, transmit diversity assisted broadband MC DS-CDMA is capable of supporting wireless communications in diverse propagation environments. Furthermore, the capacity improvement achievable by broadband MC DS-CDMA systems is also investigated. Let an overview first embark on a rudimentary of the above three CDMA schemes.

\subsection{CDMA system}

Multicarrier CDMA schemes can be broadly categorized into two groups. The first type spreads the original data stream using a spreading code and then modulates different carriers with each chip. This is usually referred to as MC-CDMA. The second type spreads the serial to parallel converted streams using a spreading code and then modulates different carriers with each data stream [21]. Denoting the bit duration as $\mathrm{Tb}$ and the chip duration as 
Tc, then the sub-carrier spacing in one system is $1 / \mathrm{Tc}$ and the other is $1 / \mathrm{Tb}$. The former is called the Multicarrier DS-CDMA (MC-DS-CDMA) and the latter is called the Multi-tone CDMA (MT-CDMA). The performance of these two schemes has been studied for an uplink channel in [29]. Hara has shown that MC-CDMA outperforms MC-DS-CDMA and MTCDMA in terms of downlink BER performance. MC-CDMA is thus an attractive technique for the downlink [30]. A simple block diagram of a MC-CDMA system is as shown below in Figure (14) below.
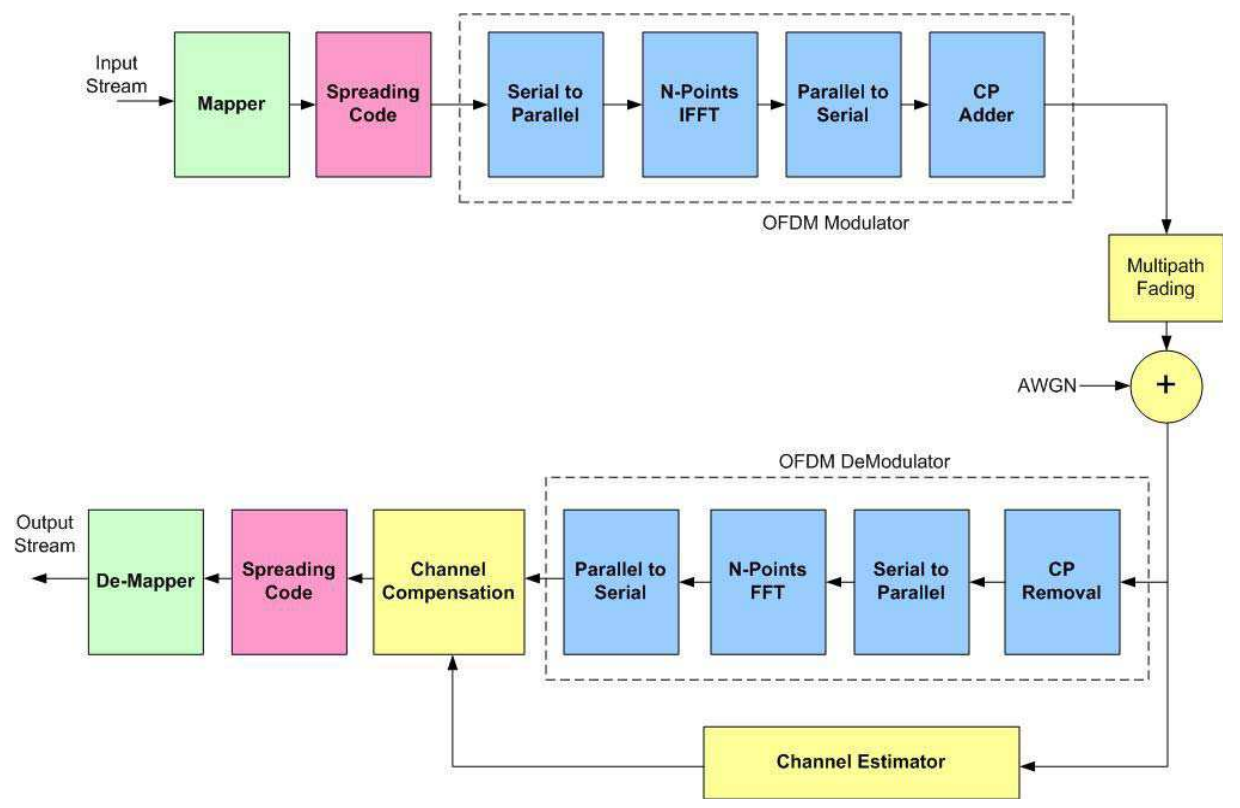

Fig. 14. Block Diagram of a FFT-MC-CDMA system

The input data stream is spread using the spreading sequence which could be a WalshHadamard code or a PN sequence. The resultant chips after spreading the symbols are modulated into different sub-carriers using the IFFT operator. The end few symbols are appended at the beginning of the frame to act as the cyclic prefix. The cyclic prefix maintains Orthogonality between the sub-carriers in a multipath channel. The receiver first removes the cyclic prefix and then performs an FFT operation of the received symbols and brings them back to the frequency domain. Then dispreading and decoding of the chips in frequency domain are performed.

\subsection{MC-DS CDMA system}

The block diagram of MC-DS CDMA transmitter is shown in Fig(15(a)) The incoming data stream is first converted to a parallel stream and then spread in time using spreading codes. This ensures that the resulting spectrum has orthogonal sub-carriers. The spreading code is represented as $C(t)$ and the processing gain is $N$. The receiver block is shown in Fig (15(b)). The dispreading is done in time after the FFT followed by a low pass filter and demodulation. The figures are adapted from [31]. 


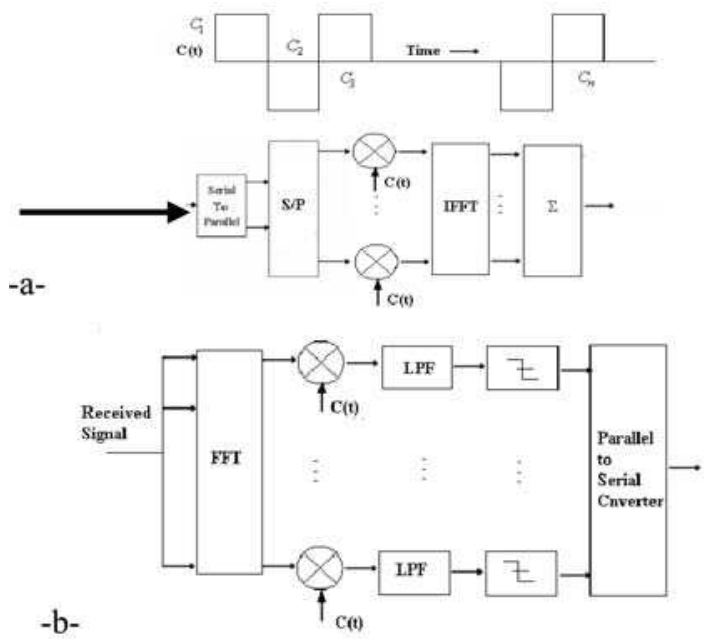

Fig. 15. (a) MC-DS CDMA Transmitter (b) MC-DS CDMA Receiver

\subsection{Multi-tone CDMA (MT-CDMA) system}

Multi-Tone CDMA transmitter spreads the serial parallel converted data streams using a spreading code in time domain so that the spreading operation can satisfy the Orthogonality condition. The MT-CDMA uses spreading codes in multiples of the number of sub-carriers as compared to MC-DS CDMA.

The transmitter block is shown in Fig (16(a)). The receiver employs Rake combining to effectively utilize the diversity due to multipath. The figures are adapted from [31,32].

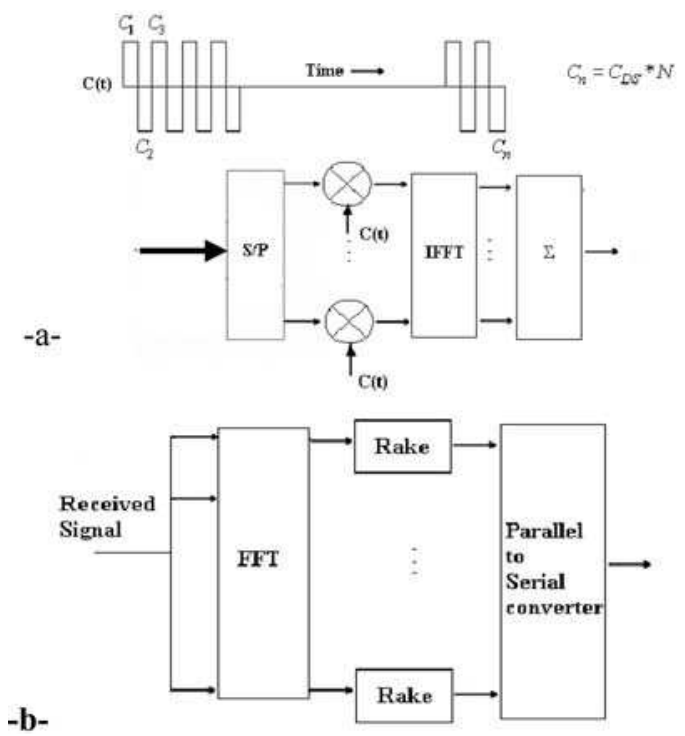

Fig. 16. (a) MT-CDMA Transmitter, (b) MT-CDMA Receiver 


\subsection{DWT based MC CDMA system}

Figure (17) below shows the block diagram of the DWT-MC-CDMA system. The only difference is that DWT is used instead of the FFT in the OFDM modulator and demodulator. Since wavelet based OFDM will not add a cyclic prefix to OFDM symbol, its data rates can surpass those of the FFT implementation.

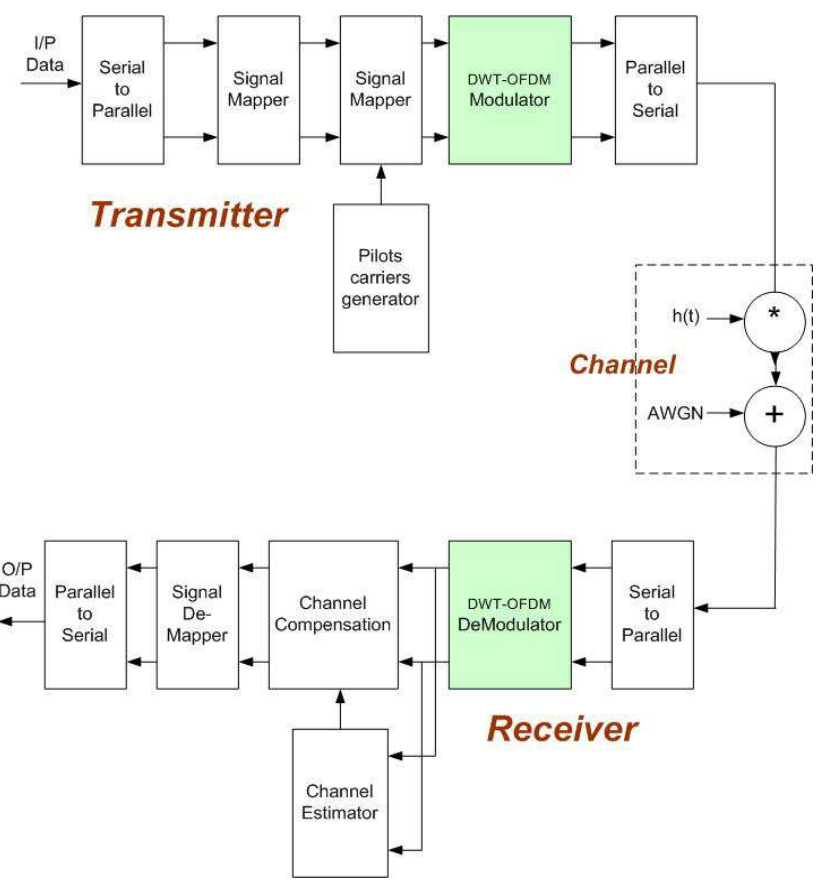

Fig. 17. Block Diagram of DWT-MC-CDMA System

\subsection{DWT based STBC-MC-DS-CDMA system}

The block diagram of the MC-DS-CDMA is depicted in Figure (18).This Figure illustrates a typical STBC-MC-DS-CDMA system used for Multicarrier modulation using one transmitter and one receiver.

\section{Simulation results}

In this section the simulation of the FFT based OFDM STBC DWTCS-OFDM system in MATLAB version 7 are achieved. And the BER performance of the OFDM system considered in different channel models, the AWGN channel, the flat fading channel. Table (1) shows the parameters of the system that are used in the simulations; the bandwidth used was $10 \mathrm{MHz}$.

\subsection{Performance of DWT-MC-CDMA in AWGN and flat fading channel models}

Simulation results of the DWT-OFDM system is shown in figure (19). It is shown clearly that the DWT-MC-CDMA is much better than the system FFT-MC-CDMA. This is a reflection to the fact that the orthogonal bases of the wavelet is much significant than the orthogonal bases used in FFT-MC-CDMA. 


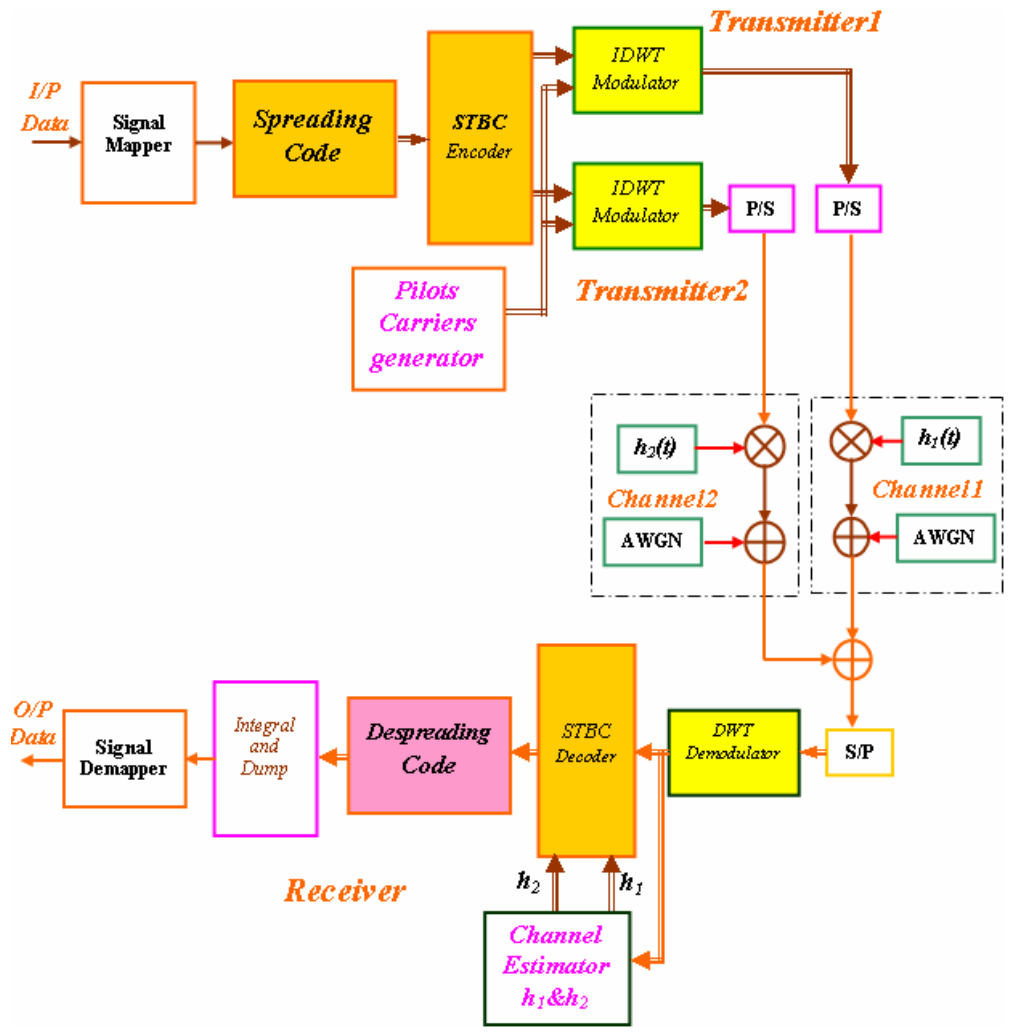

Fig. 18. Block Diagram of the DWT based STBC-MC-DS-CDMA System

\begin{tabular}{|l|l|}
\hline Modulation Types & QPSK \\
\hline Number of sub-carriers & 64 \\
\hline Number of DMWT points & 64 \\
\hline \multirow{4}{*}{ Channel model } & AWGN \\
\cline { 2 - 2 } & Flat fading+ AWGN \\
\cline { 2 - 2 } & Frequency selective fading +AWGN \\
\hline
\end{tabular}

Table 1. Simulation Parameters

Figure (20) below shows simulation result of the DWT-MC-CDMA system in the flat fading channel that assumed all the frequency components of the transmitted signal are changed correlated in phase and magnitude. Maximum Doppler Shift taken was $5 \mathrm{~Hz}$.

It is shown that wavelet based MC-CDMA is still better than that based on FFT transform. The wavelet based MC-CDMA system has $B E R=10^{-3}$ at $S N R=25 \mathrm{~dB}$, while in case of FFT based MC-CDMA system, the $B E R=10^{-3}$ achieved at approximately $\mathrm{SNR}=40 \mathrm{~dB}$, which means a gain of about $15 \mathrm{~dB}$ has been achieved. 


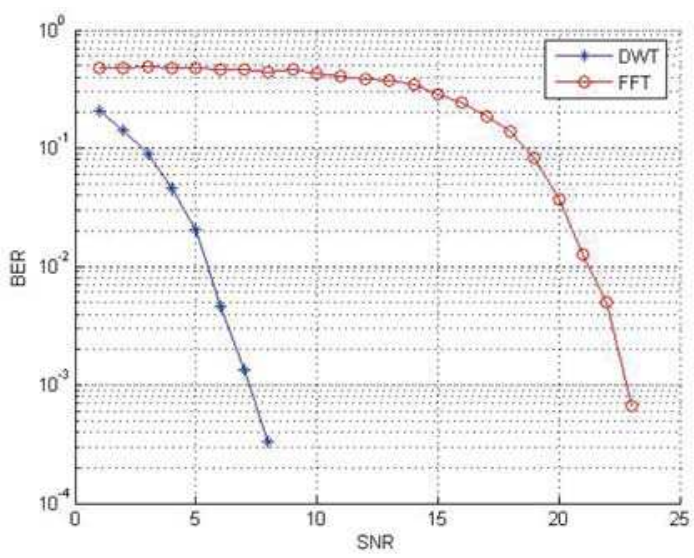

Fig. 19. BER performance of DMWT-OFDM in AWGN channel model

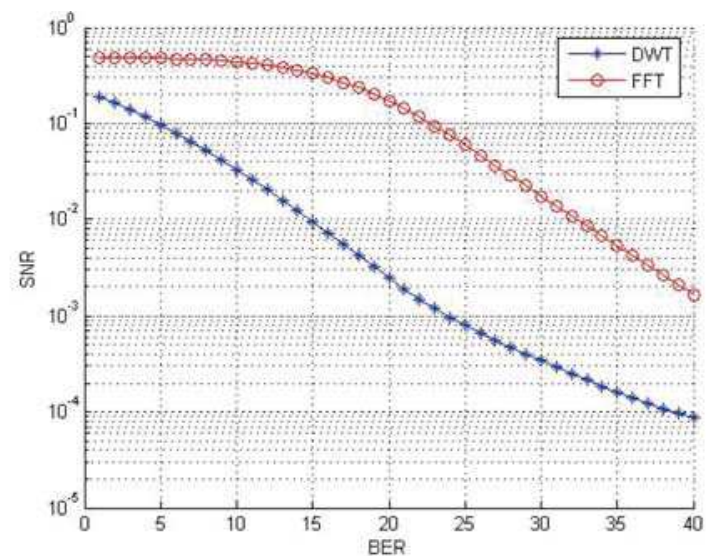

Fig. 20. BER performance of DWT-MC-CDMA in Flat Fading Channel at Max. Doppler Shift $=5$

\subsection{Performance of STBC-MC-DS-CDMA systems in AWGN and flat fading channel models}

Simulation result of the STBC-MC-DS-CDMA Systems in AWGN channel is shown in Figure (21). It is clearly shown that the STBC-MC-DS-CDMA Based on DWT is much better than STBC-MC-DS-CDMA systems Based on FFT.

In flat Fading Channel simulation a Doppler frequency of $10 \mathrm{~Hz}$ is used. From Figure (22) it can be seen that for BER=10-4 the SNR required for DWT based STBC-MC-DS-CDMA was about $13 \mathrm{~dB}$ and for FFT based STBC-MC-DS-CDMA has $25 \mathrm{~dB}$, therefore a gain of $12 \mathrm{~dB}$ for the DWT against FFT is achieved. 


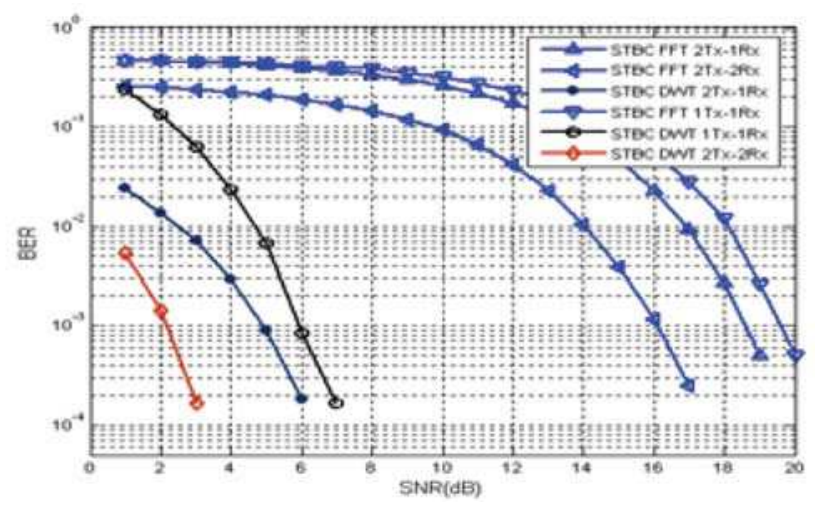

Fig. 21. Performance of STBC-MC-CDMA System in AWGN Channel.

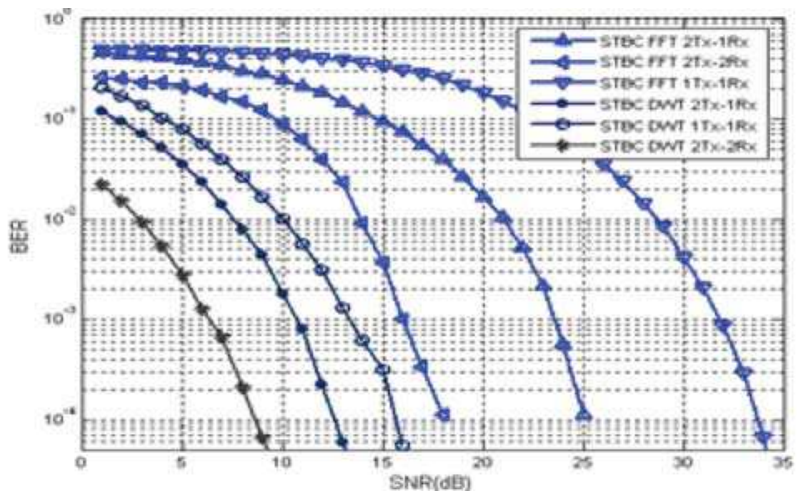

Fig. 22. Performance of S TBC-DS-CDMA in Flat Fading Channel Max. Doppler Shift $=10 \mathrm{~Hz}$.

\section{Conclusions}

The improved performance of MC-DS-CDMA system using STBC schemes and DWT is investigated. The performance comparisons of BER performance for the conventional MCDS-CDMA based on FFT, STBC MC-DS-CDMA and DWT based STBC MC-DS-CDMA in the different channel models together with their comparison for best achievable BER have been presented. Simulation results were provided to demonstrate that significant gains can be achieved by introducing such combination technique with very little decoding complexity. Therefore, the DWT based STBC MC-DS-CDMA is a feasible way to reach the next generation of wireless communication for large data rates and applications

\section{References}

[1] Shinsuke Hara, and Ramjrr Prasad. "Multicarrier Techniques for 4G Mobile Communications," Artech House, Boston. London, (2003). 
[2] Sttot J. H. "Explaining Some of the Magic COFDM," Proceedings of 20th International Television Symposium, (1997).

[3] Chuang J., and Sollenberger N. "Beyond 3G Wideband Wireless Data Access Based on OFDM and Dynamic Packet Assignment," IEEE Communication Magazine, vol. 38, no.7, pp. 78-87, July (2000)

[4] Kaiser S. "On the Performance of Different Detection Techniques for OFDM-CDMA in Fading Channels," IEEE ICC'95. pp. 2059-2063, June (1995).

[5] C. Valens, “A Really Friendly Guide to Wavelets", 1999.

[6] A. Graps, "An Introduction to Wavelets", IEEE Computational Science and Eng., Vol. 2, No. 2, 1995.

[7] Goswami J. C., Chan A. K., "Fundamentals of Wavelets Theory, Algorithms and Applications", John Wiley \& Sons Ltd. 1999.

[8] Mallat S., "A Theory for Multiresolution Signal Decomposition: the Wavelet Representation," IEEE Pattern Anal. And Machine Intel, vol. 11, no. 7, pp. 674-693. 1989.

[9] V. Strela, G. Strang et al, "The Application of Multiwavelet Filter Banks to Image Processing" IEEE Transaction on Image Processing, 1993.

[10] V. Strela, "Multiwavelets: Theory and Application", Ph.D Thesis, MIT, June 1996.

[11] H. Steendam and M. Moeneclaey "The Effect of Carrier Frequency Offsets on Downlink and Uplink MC-DS-CDMA", IEEE JOURNAL on Select. Areas in Comm., vol. 19, no. 12, Dec. 2001.

[12] S. Hara and R. Prasad "Multi-Carrier Techniques for 4G Mobile Communications", 1st Edition, Artech House, Boston, 2003

[13] Hanzo L. et al, "OFDM and MC-CDMA for Broadband Multi-User Communications, WLANs and Broadcasting," John Wiley \& Sons, (2003):

CS-Books@wiley.co.uk

[14] J. G. Proakis, “Digital Communications”, Prentice-Hall, 4th edition, 2004.

[15] Minn H., Bhargava V.K., "An Investigation into Time Domain Approach for OFDM Channel Estimation" IEEE Transaction on Broadcasting, Vol. 46, Dec 2000.

[16] Y. Zigang, and et al, "Blind Bayesian Multiuser Receiver for Space-time Coded MCCDMA System over Frequency-Selective Fading Channel", IEEE Trans. Veh. Techn, vol. VT-40, pp. 781-785, May 2001.

[17] M. Alard and R. Lassalle, "Principles of Modulation and Channel Coding for Digital Broadcasting for Mobile Receiver," Tech. Rep., no. 224, pp.47-69, Aug. 1987.

[18] P. Frederik and L. Geert "Space-Time Block Coding for Single-Carrier Block Transmission DS-CDMA Downlink" IEEE Journal On Selected Areas In Communications, Vol. 21, No. 3, pp. 350-361, APRIL 2003

[19] Hui Liu and Hujun Yin, "Receiver Design in Multi-carrier Direct-Sequence CDMA Communications", IEEE Trans. On Comm., vol. 49, no. 8, Aug. 2001.

[20] H. Steendam and M. Moeneclaey "The Effect of Carrier Frequency Offsets on Downlink and Uplink MC-DS-CDMA", IEEE JOURNAL on Select. Areas in Comm., vol. 19, no. 12, Dec. 2001.

[21] J. D. Gibson, "The Communication Handbook" 2nd Edition, Southern Methodist University Dallas, Texas, 2002. 
[22] L. Hanzo, C.H. Wong, M.S. Yee, “Adaptive Wireless Transceivers Turbo-Coded, TurboEqualized and Space-Time Coded TDMA, CDMA and OFDM Systems," John Wiley \& Sons Ltd, 2002.

[23] O.M Mustaf "Performance Evaluation of a Proposed MC-DS-CDMA for Broadband Wireless Access", PhD Thesis, University of Baghdad, 2006

[24] I. Barhumi et al, "Optimal Training Design for MIMO OFDM Systems in Mobile Wireless Channels", IEEE Trans. On Signal Processing, Vol.51, no. 6, June. 2003.

[25] Z. Cao et al "Efficient Structure-based Carrier Frequency Offset Estimation for Interleaved OFDMA Uplink", under publication of IEEE.

[26] F. Molisch “Wideband Wireless Digital Communications", 2nd Edition, Prentice Hall, New York, 2002.

[27] N.Yuan, "An Equalization Technique for High Rate OFDM Systems" M.Sc. Thesis University of Saskatchewan .Saskatoon, Dec.2003.

[28] Zhi Zliang and Li Guoqing, "A Novel Decoding Algorithm of STBC for CDMA Receiver in Multipath Fading Environments," IEEE Trans. on Comm., vol. 49, pp. 1956-1959, April 2001

[29] I. Barhumi et al, "Time-Varying FIR Equalization for Doubly-Selective Channels", IEEE Trans. On Wireless Comm., Vol. 4, no. 1, Jan. 2005.

[30] K. Ming and T. Chee "Hybrid OFDM-CDMA: A Comparison of MC/DS-CDMA, MCCDMA and OFCDM" Dept of Electrical \& Electronic, Adelaide University, SA 5005, Australia. 2002.

[31] J. Tang, and Xi Zhang, "Transmit Selection Diversity With Maximal-Ratio Combining for Multicarrier DS-CDMA Wireless Networks Over Nakagami-m Fading Channels", IEEE Journal On Selected Areas In Communications, VOL. 24, NO. 1, pp. 5710-5713, January 2006.

[32] Y. Jing "Space-Time Code Design and Its Applications in Wireless Networks" Ph.D. thesis in California Institute of Technology Pasadena, California, September 7, 2004 


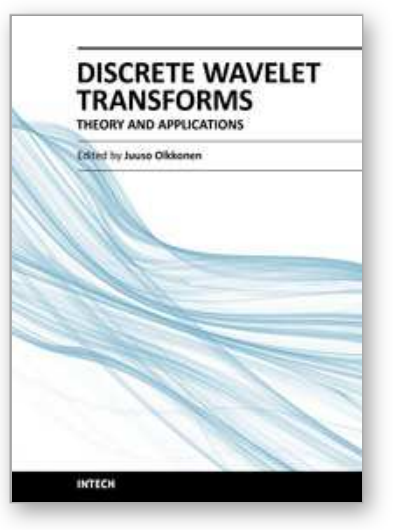

\author{
Discrete Wavelet Transforms - Theory and Applications \\ Edited by Dr. Juuso T. Olkkonen
}

ISBN 978-953-307-185-5

Hard cover, 256 pages

Publisher InTech

Published online 04, April, 2011

Published in print edition April, 2011

Discrete wavelet transform (DWT) algorithms have become standard tools for discrete-time signal and image processing in several areas in research and industry. As DWT provides both frequency and location information of the analyzed signal, it is constantly used to solve and treat more and more advanced problems. The present book: Discrete Wavelet Transforms: Theory and Applications describes the latest progress in DWT analysis in non-stationary signal processing, multi-scale image enhancement as well as in biomedical and industrial applications. Each book chapter is a separate entity providing examples both the theory and applications. The book comprises of tutorial and advanced material. It is intended to be a reference text for graduate students and researchers to obtain in-depth knowledge in specific applications.

\title{
How to reference
}

In order to correctly reference this scholarly work, feel free to copy and paste the following:

Ali A. A. (2011). Discrete Wavelet Transform Based Wireless Digital Communication Systems, Discrete Wavelet Transforms - Theory and Applications, Dr. Juuso T. Olkkonen (Ed.), ISBN: 978-953-307-185-5, InTech, Available from: http://www.intechopen.com/books/discrete-wavelet-transforms-theory-andapplications/discrete-wavelet-transform-based-wireless-digital-communication-systems

\section{INTECH}

open science | open minds

\section{InTech Europe}

University Campus STeP Ri

Slavka Krautzeka 83/A

51000 Rijeka, Croatia

Phone: +385 (51) 770447

Fax: +385 (51) 686166

www.intechopen.com

\section{InTech China}

Unit 405, Office Block, Hotel Equatorial Shanghai

No.65, Yan An Road (West), Shanghai, 200040, China

中国上海市延安西路65号上海国际贵都大饭店办公楼 405 单元

Phone: +86-21-62489820

Fax: $+86-21-62489821$ 
(C) 2011 The Author(s). Licensee IntechOpen. This chapter is distributed under the terms of the Creative Commons Attribution-NonCommercialShareAlike-3.0 License, which permits use, distribution and reproduction for non-commercial purposes, provided the original is properly cited and derivative works building on this content are distributed under the same license. 